\title{
Power-Efficient Direct-Voting Assurance for Data Fusion in Wireless Sensor Networks
}

\author{
Hung-Ta Pai and Yunghsiang S. Han
}

\begin{abstract}
Wireless sensor networks place sensors into an area to collect data and send them back to a base station. Data fusion, which fuses the collected data before they are sent to the base station, is usually implemented over the network. Since the sensor is typically placed in locations accessible to malicious attackers, information assurance of the data fusion process is very important. A witness-based approach has been proposed to validate the fusion data. In this approach, the base station receives the fusion data and "votes" on the data from a randomly chosen sensor node. The vote comes from other sensor nodes, called "witnesses," to verify the correctness of the fusion data. Because the base station obtains the vote through the chosen node, the chosen node could forge the vote if it is compromised. Thus, the witness node must encrypt the vote to prevent this forgery. Compared with the vote, the encryption requires more bits, increasing transmission burden from the chosen node to the base station. The chosen node consumes more power. This work improves the witness-based approach using direct voting mechanism such that the proposed scheme has better performance in terms of assurance, overhead, and delay. The witness node transmits the vote directly to the base station. Forgery is not a problem in this scheme. Moreover, fewer bits are necessary to represent the vote, significantly reducing the power consumption. Performance analysis and simulation results indicate that the proposed approach can achieve a 40 times better overhead than the witness-based approach.
\end{abstract}

\section{Index Terms}

wireless sensor networks, data fusion assurance, power-efficient, voting mechanism, witness

The preliminary result of this work is presented at the IEEE International Conference on Sensor Networks, Ubiquitous, and Trustworthy Computing (SUTC2006), Taiwan, R.O.C.. This work was supported by the National Science Council of Taiwan, R.O.C., under grants NSC 94-2213-E-305-002 and NSC 94-2213-E-305-001.

The authors are with the Graduate Institute of Communication Engineering, National Taipei University, Taiwan (E-mail: htpai@mail.ntpu.edu.tw; yshan@mail.ntpu.edu.tw). 


\section{INTRODUCTION}

Wireless sensor networks (WSNs) comprise many tiny, low-cost, battery-powered sensors in a small area [1], [5], [11]-[13], [21], [22]. The sensors detect environmental variations and then transmit the detection results to other sensors or a base station [2], [4], [6], [7], [24]. One or several sensors then collect the detection results from other sensors. The collected data must be processed by the sensor to reduce the transmission burden before they are transmitted to the base station. This process is called data fusion and the sensor performing data fusion is the fusion node. The fusion data may be sent from the fusion node to the base station through multiple hops [10] or a direct link [19].

Although fusion significantly lowers the traffic between the fusion node and the base station, the fusion node is more critical and vulnerable to malicious attacks than non-fusion sensors [15], [16], [20]. If a fusion node is compromised, then the base station cannot ensure the correctness of the fusion data sent to it. This problem of fusion data assurance arises because the detection results are not sent directly to the base station, so the fusion result cannot usually be verified.

This problem can be resolved in two ways: One is hardware-based [3], [14] and the other is software-based [8], [9], [17]. Since the hardware-based approach requires extra circuits to detect or frustrate the compromised node, the cost and continual power consumption of sensors are increased but still cannot guarantee protection against all attacks. Conversely, the software methods generally require no or little extra hardware for data assurance. However, as mentioned in [8], [17], several copies of the fusion data must be sent to the base station, the power consumption for the data transmission is very high.

The witness-based approach presented by Du et al. [9] does not have this difficulty. Several fusion nodes are used to fuse the collected data and have the ability to communicate with the base station. Only one node is chosen to transmit the fusion result to the base station. The other fusion nodes, serving as witnesses, encrypt the fusion results to message authentication codes (MACs). The MACs are then sent to the base station through the chosen fusion node. Finally, the base station utilizes the received MACs to verify the received fusion data. The verification could 
be wrong since the chosen node could be compromised and forge MACs. The correctness of the verification depends not only on the number of malicious fusion nodes, but also on the length of the MAC. A long MAC increases the reliability of the verification. However, the transmission of the long MAC imposes a high communication burden. If the received fusion result at the base station cannot pass the verification, then a polling scheme is started to determine whether any valid fusion result is available at the other fusion nodes. In addition to the fusion result sent by the malicious fusion node, several copies of the correct fusion result may also have to be transmitted to the base station. The transmission of the correct fusion result consumes the power of the uncompromised fusion node.

This work develops a new data fusion assurance to improve the witness-based method of Du et al. [9]. The correctness of the verification in the proposed scheme depends only on the number of compromised fusion nodes. As in the witness-based approach, a fusion node is selected to transmit the fusion result, while other fusion nodes serve as witnesses. Nevertheless, the base station obtains votes contributing to the transmitted fusion result directly from the witness nodes. No valid fusion data are available if the transmitted fusion data are not approved by a pre-set number of witness nodes. Based on this voting mechanism, two schemes are addressed: one needs variant rounds of voting and the other needs only one round of voting. In the variantround scheme, only one copy of the correct fusion data provided by one uncompromised fusion node is transmitted to the base station. Analytical and simulation results reveal that the proposed scheme is up to 40 times better on the overhead than that of $\mathrm{Du}$ et al. [9]. In the one-round scheme, the base station polls each sensor once at most. The maximum delay of the one-round scheme is much less than the variant-round scheme.

The remainder of this work is organized as follows. Section $\amalg$ briefly addresses the problem of data fusion assurance in WSNs and previous works on the problem. Section III describes the variant-round scheme and analyzes its performance in terms of overhead and delay. The description of the one-round scheme is also given. Section IV presents a performance evaluation of the proposed approach. Concluding remarks and suggestions for future work are presented in 


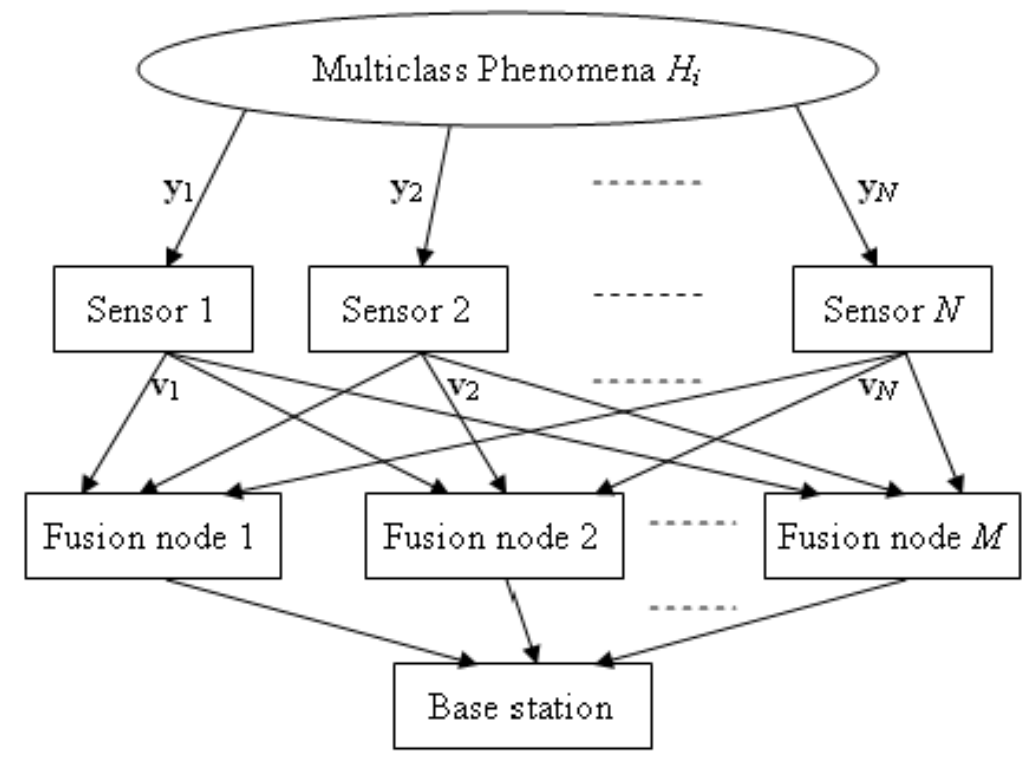

Fig. 1. Structure of a wireless sensor network for distributed detection using $N$ sensors and $M$ fusion nodes

Section $\mathrm{V}$,

\section{Data Fusion Assurance Problem and The Previous Work}

Figure 1 depicts a wireless sensor network for distributed detection with $N$ sensors for collecting environment variation data, and a fusion center for making a final decision of detections. This network architecture is similar to the so-called SENsor with Mobile Access (SENMA) [23], [28], Message Ferry [29], and Data Mule [18]. At the $j$ th sensor, one observation $y_{j}$ is undertaken for one of phenomena $H_{i}$, where $i=1,2, \ldots, L$. If the detection (raw) data are transmitted to the fusion nodes without any processing, then the transmission imposes a very high communication burden. Hence, each sensor must make a local decision based on the raw data before transmission. The decisions $1, v_{j}, j=1,2, \ldots, N$, can be represented with fewer symbols than the raw data. The sensor then transmits the local decision to $M$ fusion nodes using broadcast. The fusion node can combine all of the local decisions to yield a final result, and directly communicate with the base station. Finally, one of the fusion nodes is specified to send the final result to the base

\footnotetext{
${ }^{1}$ These decisions could be compressed data whose sizes depend on the applications of the WSNs.
} 
station. Unless all of the fusion nodes or all of the sensors fail, this detection and fusion scheme can guarantee that the base station can obtain the detection result. However, the accuracy of the result is not certain.

Two problems must be solved to ensure that the base station obtain the correct result. First, every fusion node must correctly fuse all of the local decisions, which also implies that all of the fusion results must be the same. Several algorithms have been proposed to deal with this problem [7], [25]-[27]. This work assumes that this problem has been solved. The second problem concerns assurance of the fusion result. The transmission between the fusion node and the base station is assumed herein to be error-free. Since some fusion nodes may be compromised, the fusion node chosen by the base station to transmit the fusion result may be one of the compromised nodes. Malicious data may be sent by the compromised node, and the base station cannot discover the compromised nodes from the normal fusion nodes since the data detected by the sensor are not sent directly to the base station. Consequently, the result obtained at the base station may be incorrect.

Du et al. [9] presented a witness-based approach to ensure the correctness of the fusion result. All fusion nodes, other than the chosen node, acts as witnesses of the transmitted fusion result. The witness nodes encrypt their own fusion results to MACs with private keys shared with the base station, and send the MACs, as "votes", to the chosen node. In the $T+1$ out of $M$ voting scheme, the chosen node collects all MACs from the witness nodes, and transmits them with its own fusion result to the base station. The base station can determine the received data whether the fusion result from the chosen node is accurate. If the fusion result is supported by at least $T$ MACs, then the base station accepts the fusion result. Normally, $T>\lfloor M / 2\rfloor$. However, although the number of compromised nodes $C<T$, the accepted fusion result is not always correct. If the chosen node is compromised, then it may forge the fusion result and the MAC. Let the size of each MAC be $k_{w}$ (bits). Since the number of the transmitted MACs is $M-1$, the number of the transmitted bits, in addition to the fusion result, is $(M-1) k_{w}$. The probability that the base 
station accepts the forged fusion result is given by

$$
P_{e}=\sum_{i=T}^{M-1}\left(\begin{array}{c}
M-1 \\
i
\end{array}\right)\left(\frac{1}{2^{k_{w}}}\right)^{i}\left(1-\frac{1}{2^{k_{w}}}\right)^{M-i-1} .
$$

For instance, consider the majority voting rule in which $T+1 \geq\lceil M / 2\rceil$. To ensure that $P_{e} \leq 2^{-10}$, set $k_{w}=\lceil 2(10 /(M-1)+1)\rceil$. Additionally, although only one copy of the fusion result is sent to the base station in this witness-based approach, the witness nodes still requires significant communication bandwidth because the MACs of the fusion results are transmitted.

To compare the proposed scheme fairly with the witness-based approach, the overhead is defined as the total number of bits, except the bits for one copy of the correct fusion result, transmitted to the base station by uncompromised nodes during the data assurance process 2 The overhead can therefore be regarded as the useful power consumed for the data assurance. Moreover, the round delay is defined as the number of rounds 3 required to collect all MACs (votes) from the witness nodes and the polling delay the number of votes (including all agree and disagree voting) 4

The overhead of the witness-based approach given in [9] is then derived as follows. If the received fusion result is not accepted, then the base station may start a polling mechanism to seek the correct fusion result. The base station randomly specifies another fusion node. The new chosen node then sends its fusion result and all MACs from the witness nodes to the base station 5 When the number of compromised fusion nodes, $C$, is greater than $M-T-1$ but less than $T+1.6$ the fusion result is invalid and $M-T$ fusion nodes are chosen to transmit their fusion results to the base station in the polling process. Since the overhead defined only considers

\footnotetext{
${ }^{2}$ The method does not consider the power consumption of all compromised nodes since they are not useful to the WSN.

${ }^{3}$ Or the number of fusion results sent to the base station.

${ }^{4}$ The overall time delay is then can be derived by these two delays.

${ }^{5}$ All MACs must be sent to the base station again to avoid the denial of service since the previously chosen compromised fusion node might modify MACs it sent to the base station. This action is not clearly presented in [9].

${ }^{6}$ The $C$ compromised nodes are assumed to collude to forge a wrong fusion result. Hence, if $C \geq T+1$, then they can successfully forge a wrong fusion result, and the base station accepts the forged result.
} 
the power consumption of uncompromised nodes, the number of uncompromised nodes among the $M-T$ fusion nodes must be discovered. The probability that $i$ of the $M-T$ fusion nodes are uncompromised is given by

$$
\begin{aligned}
P_{w}(i) & =\frac{\left(\begin{array}{c}
M-C \\
i
\end{array}\right)\left(\begin{array}{c}
C \\
M-T-i
\end{array}\right)}{\left(\begin{array}{c}
M \\
M-T
\end{array}\right)} \\
= & \frac{\left(\begin{array}{c}
M-T \\
i
\end{array}\right)\left(\begin{array}{c}
T \\
M-C-i
\end{array}\right)}{\left(\begin{array}{c}
M \\
C
\end{array}\right)}
\end{aligned}
$$

where $0 \leq i \leq M-T$. Let $K$ be the number of bits representing the fusion result. The average overhead is thus

$$
O_{w}(M, T, C)=\sum_{i=1}^{M-T} P_{w}(i)\left[(M-1) i k_{w}+K(i-1)\right] \text { (bits) }
$$

where $i-1$ is used because the overhead is defined such that one copy of correct fusion result does not count on. Equation (1) indicates that the number of the correct fusion results transmitted by the uncompromised fusion nodes may be up to $M-T$. In other words, the power of the uncompromised nodes is significantly wasted. Moreover, because each chosen node has to collects all MACs from the witness nodes, the average round delay, $R_{w}$, is $M-T$ and the average polling delay $D_{w}$ is $(M-T)(M-1)$.

Conversely, when the number of uncompromised nodes is greater than $T$, the base station can obtain the correct fusion result. If the base station gets the correction result at round $i$, meaning that the chosen fusion nodes from round 1 to $i-1$ are compromised, then the average round 
delay can be given by

$$
R_{w}=\sum_{i=1}^{C+1} i \frac{\left(\begin{array}{c}
M-i \\
C-i+1
\end{array}\right)}{\left(\begin{array}{c}
M \\
C
\end{array}\right)}
$$

and the average polling delay is $R_{w}(M-1)$. The average overhead is given by $(M-1) k_{w}$. Notice that the maximum round delay is $C+1$. When the fusion result is valid, 40 and 60 bits must be transmitted to the base station when $M=11\left(k_{w}=4\right.$, i.e., $\left.P_{e} \leq 2^{-10}\right)$ and $M=21$ $\left(k_{w}=3\right.$, i.e., $P_{e} \leq 2^{-10}$ ), respectively, setting $K=0$ (in practice, $K>0$ ). A large amount of power must be consumed for this transmission, significantly reducing the lifetime of the fusion node. The problem of power consumption is even worse when the fusion result is invalid. For example, the maximum average overhead is about 109 and 314 bits for $M=11(C=5$ and $T=6)$ and $M=21(C=10$ and $T=11)$, respectively. Therefore, the witness-based approach must be enhanced.

\section{IMPROVED VOTING MECHANISM}

The voting mechanism in the witness-based approach is designed according to the MAC of the fusion result at each witness node. This design is reasonable when the witness node does not know about the fusion result at the chosen node. However, in practice, the base station can transmit the fusion result of the chosen node to the witness or the witness node is in the communication range of the chosen node and the base station. Therefore, the witness node can obtain the transmitted fusion result from the chosen node through the base station or overhearing. The witness node then can compare the transmitted fusion result with its own fusion result. Finally, the witness node can send its vote (agreement or disagreement) on the transmitted result directly to the base station, rather than through the chosen node.

The base station has to set up a group key for all fusion nodes to ensure that the direct voting 
mechanism works 7 When a fusion node wishes to send its fusion result to the base station, it adopts the group key to encrypt the result, and other fusion nodes serving as witness nodes can decode the encrypted result. The witness node then starts to vote on the transmitted result. Two data fusion assurance schemes are proposed based on the voting mechanism using a group key.

\section{A. Variant-round Scheme}

In this scheme, the base station needs to ask the witness node whether it agrees or disagrees with the transmitted fusion result. The witness node then sends its vote to the base station. If the transmitted fusion result is not supported by at least $T$ witness nodes, then the base station might have to select a witness node that does not agree with the transmitted result as the next chosen node. The detail steps of the scheme are given as follows:

Step 1: The base station chooses a fusion node. Other fusion nodes serve as witness nodes. Define a set of witness nodes that includes all witness nodes and let the nodes in the set be randomly ordered. Denote $M^{\prime}=M-1$ as the size of the witness set in the current round.

Step 2: The chosen node transmits its fusion result to the base station.

Step 3: The base station polls the node in the witness set by following the order of the witness nodes. The polling-for-vote 8 process does not stop until

- $T$ witness nodes agree with the transmitted fusion result (agreeing nodes), where $1 \leq T \leq M-1$

- $M^{\prime}-T+1$ witness nodes disagree with the transmitted fusion result (disagreeing nodes), or

- all witness nodes have been polled.

\footnotetext{
${ }^{7}$ We assume that all witnesses can overhear the fusion result sent by the chosen node. If it is not the case, the direct voting mechanism needs to be slightly modified.

${ }^{8}$ The rest of this work utilizes "voting", "polling" and "polling-for-vote" interchangeably.
} 
Step 4: Represent $A$ as the number of witness nodes that agree with the transmitted fusion result.

- If $A=T$, then the transmitted fusion result passes the verification of the fusion result. Stop the polling.

- If $M^{\prime}-T-1<A<T$, then no reliable fusion result is valid. Stop the polling.

- If $A \leq M^{\prime}-T-1$, then exclude the $A$ agreeing witness nodes from the witness set. Let the first node that disagrees with the transmitted fusion result be the chosen node to transmit its fusion result. Thus, the updated size of the witness set, $M^{\prime}$, becomes to $M^{\prime}-A-19$. Go to Step 2 for the next round of the polling.

\section{B. Analysis of the Variant-round Scheme}

This analysis assumes that the compromised node always transmits the forged fusion result while the compromised node is chosen to send its fusion result. When a compromised node serves as a witness node, it always disagrees with the correct fusion result, and agrees with the forged fusion result with a probability $P_{f}$. If the compromised node attempts to make the base station accept the forged fusion result, then it always agrees with the fusion result transmitted by other compromised nodes, i.e., $P_{f}=1$, and at most two rounds of voting have to be run. Conversely, if the compromised node wants to make the polling-for-vote process run as long as possible, then it always disagrees with the transmitted fusion result, i.e., $P_{f}=0$. The performance of the variant-round scheme when $P_{f}=0$ is analyzed next. Appendix describes the performance analysis of the variant-round scheme when $P_{f}=1$. When $P_{f} \neq 0,1$, the overheads are presented by computer simulations in next section. Since all compromised nodes (uncompromised nodes) have the same behavior in the following analysis, the analysis can be treated as the problem of counting for $C$ black balls (compromised nodes) and $M-C$ white balls (uncompromised nodes) together.

\footnotetext{
${ }^{9}$ The number of nodes performing the polling-for-vote at next round becomes to $M^{\prime}-A$.
} 
If the compromised node always disagrees with the transmitted fusion result, then no forged fusion result is accepted. Two cases must be addressed:

Case $1 C \geq M-T$,

Case $2 C<M-T$.

Note that the valid fusion result is not available in Case 1.

Assume that the chosen node at the first round is compromised. The probability that the chosen node is compromised at the first round is given by $C / M$. The first-round voting finishes when $M-T$ witness nodes do not agree with the transmitted fusion result, as described in Step 3. Thus, the polling order (i.e., the order of witness nodes as described in Step 1) determines the number of witness nodes that the base station has to poll at this round of voting. The number of possible polling orders in the sense of the black-white-ball model is given by

$$
\Pi_{v 1}^{c 1}=\frac{(M-1) !}{(C-1) !(M-C) !}=\left(\begin{array}{c}
M-1 \\
C-1
\end{array}\right),
$$

where the subscript, $v 1$, denotes the first case of the variant-round scheme, and the superscript, $c 1$, represents the first round of voting when the chosen node is compromised. Since the chosen node at the first-round voting is compromised, the polling stops after $M-T$ witness nodes have been polled. Moreover, the number of unpolled nodes is $M-1-(M-T)=T-1$, and the number of uncompromised nodes among the unpolled nodes is $M-C-i$ if there are $i$ uncompromised nodes polled. Thus, the probability that $i$ of the $M-T$ polled nodes are uncompromised, where $0 \leq i \leq M-T, 10$ is then written by

$$
\frac{1}{\Pi_{v 1}^{c 1}}\left(\begin{array}{c}
M-T \\
i
\end{array}\right)\left(\begin{array}{c}
T-1 \\
M-C-i
\end{array}\right) .
$$

No node is excluded from the witness set. The number of compromised nodes and the size of the witness set at the second round become $C-1$ and $M-2$, respectively. The average overhead,

${ }^{10}$ Actually, $\max \{M-T-C+1,0\} \leq i$. Since $\left(\begin{array}{l}a \\ b\end{array}\right)$ is 0 when $b>a, 0$ is adopted as the lower bound of $i$. 
when the chosen node at the first round is compromised, is expressed recursively by

$$
O_{v 1}^{c}(M, T, C, 0)=\sum_{i=0}^{M-T} \frac{1}{\Pi_{v 1}^{c 1}}\left(\begin{array}{c}
M-T \\
i
\end{array}\right)\left(\begin{array}{c}
T-1 \\
M-C-i
\end{array}\right) i k^{\prime}+O_{v 1}(M-1, T, C-1,0),
$$

where $O_{v 1}(M-1, T, C-1,0)$ represents the average overhead when the number of fusion nodes is $M-1$ and the number of compromised nodes is $C-1$, and $k\left(k^{\prime}\right)$ is the number of bits that a witness must send to the base station while it agrees (disagrees) with the transmitted fusion result 11 Moreover, the average round delay and the average polling delay under the same condition are represented by

$$
R_{v 1}^{c}(M, T, C, 0)=1+R_{v 1}(M-1, T, C-1,0),
$$

and

$$
D_{v 1}^{c}(M, T, C, 0)=M-T+D_{v 1}(M-1, T, C-1,0)
$$

where $R_{v 1}(M-1, T, C-1,0)$ and $D_{v 1}(M-1, T, C-1,0)$ are the average round delay and polling delay, respectively, when the number of fusion nodes performing the polling-for-vote is $M-1$ and the number of compromised nodes among them is $C-1$.

Next, suppose that the chosen node at the first round is not compromised, which has a probability of $(M-C) / M$. The number of the possible polling orders is given by

$$
\Pi_{v 1}^{u 1}=\left(\begin{array}{c}
M-1 \\
C
\end{array}\right)
$$

where the superscript, $u 1$, denotes the first round of polling while the chosen node is uncompromised. When the polling stops at witness node $j$, the node does not agree with the transmitted result and the base station has polled $M-T$ disagreeing nodes (including witness node $j$ ). Moreover, $M-j-1$ nodes are unpolled, and $C-(M-T)=T+C-M$ of these are compromised. Since the witness set has $M-C-1$ uncompromised nodes, the maximum number

\footnotetext{
${ }^{11}$ The bits sent to the base station when a node agrees with the fusion result are separated from those sent when the node disagrees with the result, since the node can be silenced when it agrees with the result, while only a few bits are sent when it disagrees.
} 
of polled witness nodes is $(M-C-1)+(M-T)$. Thus, the probability that the polling stops at the $j$ th witness node, where $M-T \leq j \leq(M-C-1)+(M-T)=2 M-T-C-1$, is given by

$$
P_{v 1}^{u 1}(j)=\frac{1}{\Pi_{v 1}^{u 1}}\left(\begin{array}{c}
j-1 \\
M-T-1
\end{array}\right)\left(\begin{array}{c}
M-j-1 \\
T+C-M
\end{array}\right) .
$$

Since the number of unpolled nodes $M-j-1$ is less than $T$, these nodes will never be polled in the following runs before the voting mechanism stops. Hence, only compromised nodes are polled after the first round and then no uncompromised nodes are further polled. Note that the number of uncompromised nodes among the polled nodes is $j-(M-T)=j-M+T$. Therefore, the average overhead, when the chosen node at the first round is not compromised, is then given by

$$
O_{v 1}^{u}(M, T, C, 0)=\sum_{j=M-T}^{2 M-T-C-1} P_{v 1}^{u 1}(j)(j-M+T) k .
$$

Moreover, the size of the witness set after the first round becomes $M^{\prime}=(M-j-1)+(M-$ $T)-1=2 M-T-j-2$ (the total number of unpolled and disagreeing nodes minus 1 ). The polling process stops if

$$
\begin{aligned}
& M^{\prime} \leq T-1 \\
\Leftrightarrow & 2 M-T-j-2 \leq T-1 \\
\Leftrightarrow & j \geq 2 M-2 T-1 .
\end{aligned}
$$

Otherwise, the size is decreased by 1 in each following round until it becomes $T$. Consequently, the total number of the following rounds is $2 M-T-j-2-T+1=2 M-2 T-j-1$ and then the number of the total rounds is $2 M-2 T-j-1+1=2 M-2 T-j$. The average round delay is then represented by

$$
R_{v 1}^{u}(M, T, C, 0)=\sum_{j=M-T}^{2 M-2 T-2} P_{v 1}^{u 1}(j)(2 M-2 T-j)+\sum_{j=2 M-2 T-1}^{2 M-T-C-1} P_{v 1}^{u 1}(j) .
$$


Since the voting process will stop when $M^{\prime}-T+1$ nodes are polled at each round, the polling delay is

$$
\begin{aligned}
D_{v 1}^{u}(M, T, C, 0) & =\sum_{j=M-T}^{2 M-T-C-1} P_{v 1}^{u 1}(j) j \\
& +\sum_{j=M-T}^{2 M-2 T-2} P_{v 1}^{u 1}(j) \frac{(2 M-2 T-j)(2 M-2 T-j-1)}{2} .
\end{aligned}
$$

Equations (2) to (7), and the initial conditions then give the average overhead and the average delays of Case 1 as

$$
\begin{aligned}
& O_{v 1}(M, T, C, 0)=\left\{\begin{array}{ll}
0 & M \leq T \\
\frac{C}{M} O_{v 1}^{c}(M, T, C, 0)+\frac{M-C}{M} O_{v 1}^{u}(M, T, C, 0) & \text { else }
\end{array},\right. \\
& R_{v 1}(M, T, C, 0)=\left\{\begin{array}{ll}
0 & M \leq T \\
\frac{C}{M} R_{v 1}^{c}(M, T, C, 0)+\frac{(M-C)}{M} R_{v 1}^{u}(M, T, C, 0) & \text { else }
\end{array},\right. \\
& D_{v 1}(M, T, C, 0)=\left\{\begin{array}{ll}
0 & M \leq T \\
\frac{C}{M} D_{v 1}^{c}(M, T, C, 0)+\frac{(M-C)}{M} D_{v 1}^{u}(M, T, C, 0) & \text { else }
\end{array} .\right.
\end{aligned}
$$

The second case, i.e., $C<M-T$, produces a valid fusion result. Similarly, if the chosen node at the first-round polling is compromised, then the polling stops after $M-T$ witness nodes are polled. The average overhead and the average delays, when the chosen node at the first round is compromised, are expressed respectively as

$$
\begin{aligned}
& O_{v 2}^{c}(M, T, C, 0)=\sum_{i=M-T-C+1}^{M-T} \frac{1}{\Pi_{v 1}^{c 1}}\left(\begin{array}{c}
M-T \\
i
\end{array}\right)\left(\begin{array}{c}
T-1 \\
M-C-i
\end{array}\right) i k^{\prime}+O_{v 2}(M-1, T, C-1,0), \\
& R_{v 2}^{c}(M, T, C, 0)=1+R_{v 2}(M-1, T, C-1,0), \\
& D_{v 2}^{c}(M, T, C, 0)=M-T+D_{v 2}(M-1, T, C-1,0) .
\end{aligned}
$$

Only one round of polling is needed when the chosen node is uncompromised at the first round. When the polling stops at witness node $j$, the node agrees with the transmitted result and the base station has polled $T$ agreeing nodes (including witness node $j$ ). Moreover, $M-j-1$ nodes are unpolled, and $M-1-C-T$ of these are uncompromised. The probability that the polling 
process ends at the $j$ th witness node, where $T \leq j \leq T+C$, is given by

$$
P_{v 2}^{u 1}(j)=\frac{1}{\Pi_{v 1}^{u 1}}\left(\begin{array}{c}
j-1 \\
T-1
\end{array}\right)\left(\begin{array}{c}
M-j-1 \\
M-C-T-1
\end{array}\right) .
$$

The number of polled uncompromised nodes is $T$. The average overhead when the chosen node is uncompromised at the first round is represented as

$$
O_{v 2}^{u}(M, T, C, 0)=\sum_{j=T}^{T+C} P_{v 2}^{u 1}(j) T k,
$$

and the average polling delay is

$$
D_{v 2}^{u}(M, T, C, 0)=\sum_{j=T}^{T+C} P_{v 2}^{u 1}(j) j .
$$

Consequently, the average overhead $O_{v 2}(M, T, C, 0)$, the average round delay $R_{v 2}(M, T, C, 0)$, and the average polling delay $D_{v 2}(M, T, C, 0)$ can be represented as

$$
\begin{aligned}
& O_{v 2}(M, T, C, 0)= \begin{cases}0 & M \leq T \\
T k & M>T \text { and } C=0 \\
\frac{C}{M} O_{v 2}^{c}(M, T, C, 0)+\frac{M-C}{M} O_{v 2}^{u}(M, T, C, 0) & \text { else }\end{cases} \\
& R_{v 2}(M, T, C, 0)= \begin{cases}0 & M \leq T \\
1 & M>T \text { and } C=0 \\
\frac{C}{M} R_{v 2}^{c}(M, T, C, 0)+\frac{M-C}{M} & \text { else }\end{cases} \\
& D_{v 2}(M, T, C, 0)= \begin{cases}0 & M \leq T \\
T & M>T \text { and } C=0 \\
\frac{C}{M} D_{v 2}^{c}(M, T, C, 0)+\frac{M-C}{M} D_{v 2}^{u}(M, T, C, 0) & \text { else }\end{cases}
\end{aligned}
$$

An interesting property of the variant-round scheme is that throughout the polling process, at most one fusion result is transmitted from all of the uncompromised nodes to the base station. Hence, the overhead of the scheme is independent of the size of the fusion result 12 This claim can be proven by the following argument. In the case of a valid fusion result, when the fusion result from an uncompromised node is sent to the base station at a round of polling, the polling

\footnotetext{
${ }^{12}$ Note that the overhead is defined as the total number of bits transmitted minus the bits of the correct fusion result.
} 
process stops at this round and the valid fusion result is obtained by the base station. Accordingly, only one valid fusion result is sent to the base station by all uncompromised nodes. In the case of no valid fusion result, the number of uncompromised nodes as witnesses is less than $T$. If a round of the polling process is the first time that the chosen node is uncompromised, this round terminates when either all witness nodes have been polled or $M^{\prime}-T+1$ witness nodes disagree with the transmitted fusion result. In the former case, the polling process stops. In the latter case, another polling round is required. Importantly, in the next round all uncompromised nodes will be the last $T-1$ nodes in the witness set and will not then be chosen to send any fusion result before the polling process is completed 13 Therefore, only one fusion result is sent by all uncompromised nodes when no valid result can be obtained by the base station.

\section{One-round Scheme}

The number of rounds in the above scheme is not fixed. Hence, the delay varies. Variant delay is not desired in some applications such as real-time systems. This work proposes another scheme that is based on the improved voting mechanism. In this scheme the base station may receive different fusion results from the witness nodes. It requires that all received fusion results be stored. This scheme has a fixed delay and is summarized as follows:

Step 1: The base station randomly chooses a fusion node. Other fusion nodes serve as witness nodes. Define a set of witness nodes that includes all of the witness nodes and let the nodes in the set be randomly ordered.

Step 2: The chosen node transmits its fusion result to the base station. The base station sets the fusion result as a potential voting result and the number of agreeing votes for the fusion result is set to be zero.

Step 3: The base station polls the nodes, with the voting result, one by one in the witness set, following the order of the witness nodes. The witness node compares its fusion result with the voting result.

\footnotetext{
${ }^{13}$ In the next round, all uncompromised polled nodes are deleted from the witness set according to the scheme.
} 
- If the witness node agrees with the voting result, it sends an agreeing vote to the base station. The base station increases the number of agreeing votes for the voting result by one.

- If the witness node does not agree with the voting result, it transmits its fusion result to the base station.

- If the fusion result has been stored in the base station, then the base station increases the number of agreeing votes for the fusion result by one.

- If the fusion result has not been stored in the base station, then the base station stores the fusion result and the number of agreeing votes for the fusion result is set to be zero.

The base station sets the voting result to the received fusion result with the maximum number of agreeing votes to poll the next witness node. If two or more fusion results received the maximum umber of votes, then the voting result is set to the result with the most recent vote. The polling stops when any received fusion result receives $T$ votes or when the number of unpolled nodes plus the maximum number of votes for the results recorded at the base station is less than $T$.

\section{Performance Evaluation}

In this section numerical and computer simulations are performed to evaluate the performance of the proposed schemes. The performances of the proposed variant-round scheme are numerically calculated by the results given in Section III when $P_{f}=0$ and 1 . The performances of the variant-round scheme when $P_{f} \neq 0$ or 1 and that of the one-round scheme are evaluated using Monte Carlo computer simulations. The proposed schemes are compared using the witness-based approach in terms of overhead, average round delay, and average polling delay performances. For the witness-based approach, the size of each MAC, $k_{w}$, is assumed to be four bits. In the evaluation of the overhead of the variant-round scheme, the size of fusion result is zero (i.e., $K=0$ ), such that the witness-based approach has the best performance. As stated at the 
end of Subsection $\amalg$ III-B, the overhead of the variant-round scheme is independent of the size of the fusion result. Under this assumption, the variant-round scheme is inferred to have better overhead performance than the witness-based approach for all sizes of the fusion result whenever it outperforms with a zero-sized the fusion result. All results are presented for number of nodes $M=1114$

Figure 2 shows the overheads of the variant-round scheme when $P_{f}=0$. As in the example in Section $\llbracket, k^{\prime}=4$ is set first and then set $k=1, k^{\prime}=0$ and $k=0, k^{\prime}=1$ are considered. This figure indicates that setting $k=1$ and $k^{\prime}=0$ significantly reduces the overhead of the proposed scheme, independently of the fusion result at the base station. Setting $k=0$ and $k^{\prime}=1$ further reduces the overhead of the proposed scheme. Next, the variant-round (VR) scheme is compared with the witness-based approach. In the variant-round scheme, $k=0$ and $k^{\prime}=1$ are set. Figure 3 compares overheads. The variant-round scheme significantly outperforms the witness-based approach regardless of the fusion result at the base station. For example, according to Fig. 3, when $T=5$ and $M=11$ the variant-round scheme is almost 40 times better than the witness-based approach given in [9] in terms of overhead performance for $C=1$ and 2 . Figure 4 compares the average round delays of the proposed variant-round scheme and the witness-based approach. This figure demonstrates that the average round delays of the proposed scheme are smaller than those of the witness-based approach when the base station can obtain the valid fusion results; however, they perform equally when the base station obtains invalid fusion results. Figure 5 compares the average polling delays of the proposed variant-round scheme and the witness-based approach. In Fig. 5, the proposed scheme has much smaller average polling delays than the witness-based approach - unlike average round delay performance. For example, when $T=10$ and $M=11$, the proposed variant-round scheme is almost five times better than the witness-based approach in terms of average polling delay performance for $C=4$ and 5 . Accordingly, the proposed scheme outperforms the witness-based approach given in [9] in terms

\footnotetext{
${ }^{14}$ Similar results can be obtained for $M=21$, but omitted because of the page limit.
} 
TABLE I

MAXIMUM AVERAGE OVERHEADS (BITS) FOR VARIANT-ROUND (VR) SCHEME AND WITNESS-BASED APPROACH GIVEN IN [9]

\begin{tabular}{|l||c|c|c|c|c|c|}
\hline & Witness-based & VR, $P_{f}=0$ & VR, $P_{f}=0.25$ & VR, $P_{f}=0.5$ & VR, $P_{f}=0.75$ & VR, $P_{f}=1$ \\
\hline$M=11$ & 109 & 2.5 & 2.5 & 2.4 & 2.7 & 3.2 \\
\hline$M=21$ & 314 & 4.9 & 4.7 & 4.7 & 4.9 & 6.2 \\
\hline
\end{tabular}

of overhead and delay.

The following computer simulations evaluate the variant-round scheme when $P_{f}=0.25,0.5$, and 0.75 by performing 10000 Monte Carlo tests for each simulation. In the first set of simulations, the witness-based approach given in [9] is compared with the proposed variant-round scheme when $P_{f}=0.25,0.5$, and 0.75 . Figure 6 presents the results for $M=11, k=0$ and $k^{\prime}=1$. The variant-round scheme outperforms the witness-based approach in every $P_{f}$ simulated. For example, in Fig. 6, when $T=5$ the proposed variant-round scheme is almost 40 times better than the witness-based approach given in [9] in terms of overhead performance for $C=1,2$ and $P_{f}=0.25,0.5,0.75$. Finally, Table 【 summarizes the maximum average overhead of the variant-round scheme and the witness-based approach for $M=11$ and 12 .

In the second set of simulations, the average numbers of bits sent by uncompromised nodes in the one-round scheme is evaluated. When the compromised node does not agree with the voting result, the fusion result transmitted by the compromised node is different from other fusion results and the size of the fusion result is $K=48$. Fig. 7 shows the results for $M=11$ when $P_{f}=0,0.5$, and 1. In Fig. 7(a), when the base station can obtain a valid fusion result, the bits transmitted by the uncompromised nodes to the base station in the one-round scheme increase with the number of compromised nodes $C$, as expected. However, they are smaller than those in the witness-based approach. Notably, in this case, the bits transmitted by uncompromised nodes in the witness-based approach is constant, since once an uncompromised node is polled, the polling process is completed. For small $C$, such as $C=0,1,2$ or 3 , the number of bits 


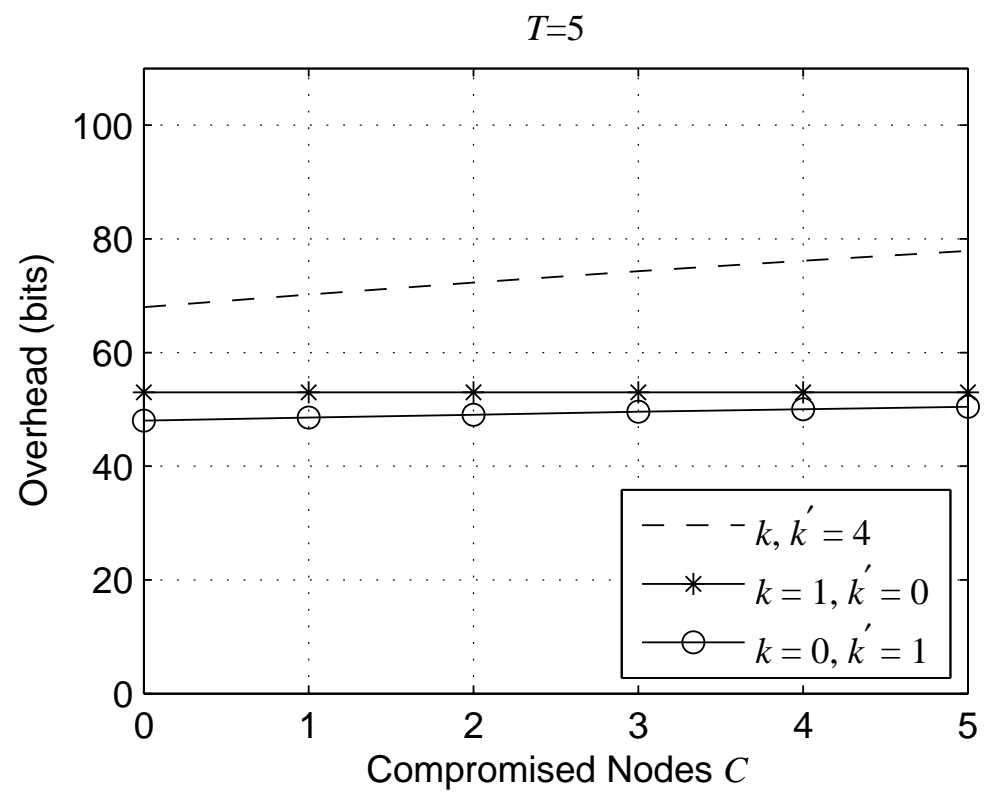

(a)

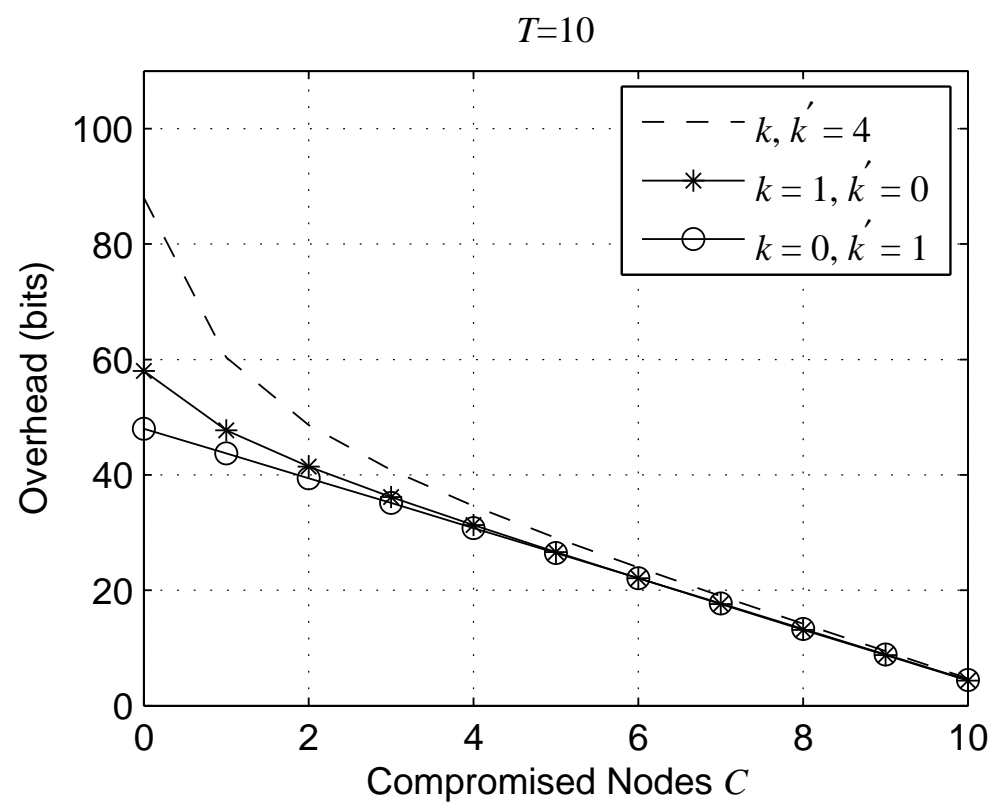

(b)

Fig. 2. The overheads of the variant-round scheme, for $M=11$ and $P_{f}=0$, (a) when $T=5$ (valid fusion result) and (b) when $T=10$ (invalid fusion result). 


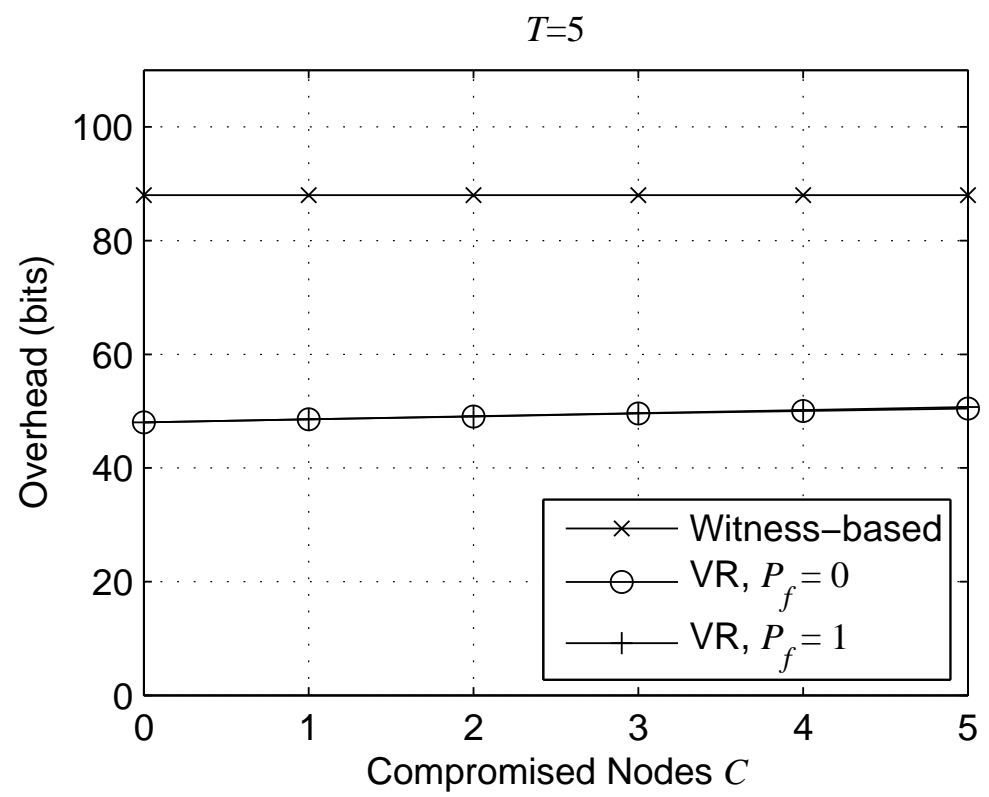

(a)

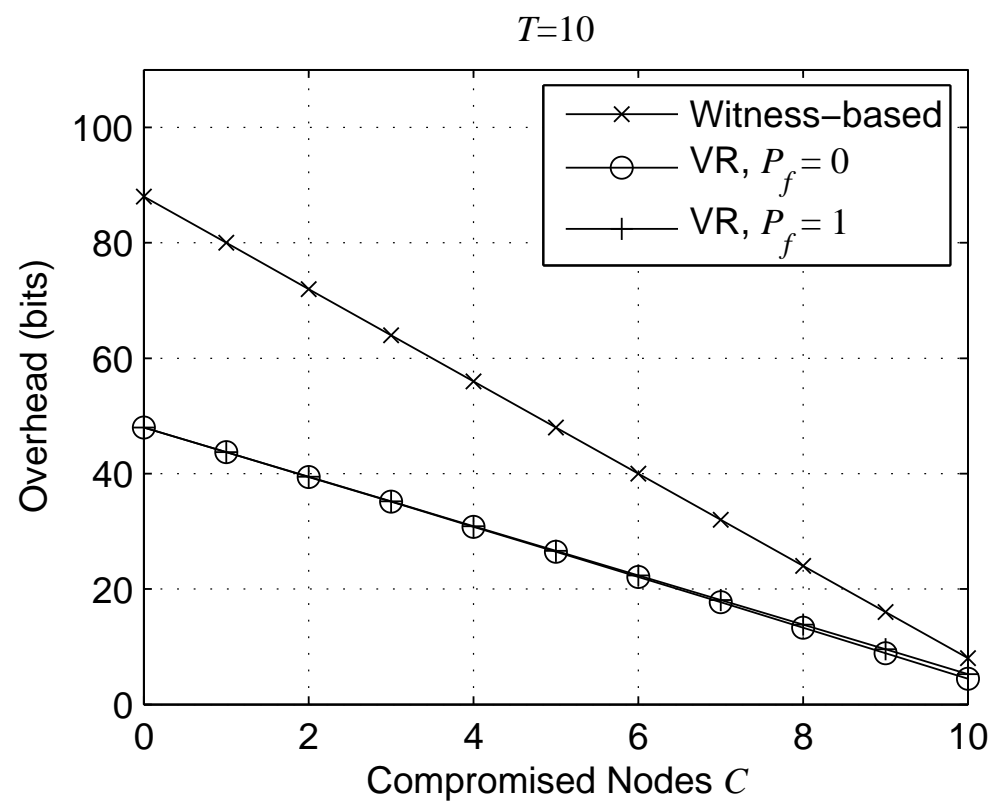

(b)

Fig. 3. Overhead comparison between the variant-round (VR) scheme and the witness-based approach given in [9], for $M=11$ and $P_{f}=1,0$, (a) when $T=5$ (valid fusion result) and (b) when $T=10$ (invalid fusion result). 


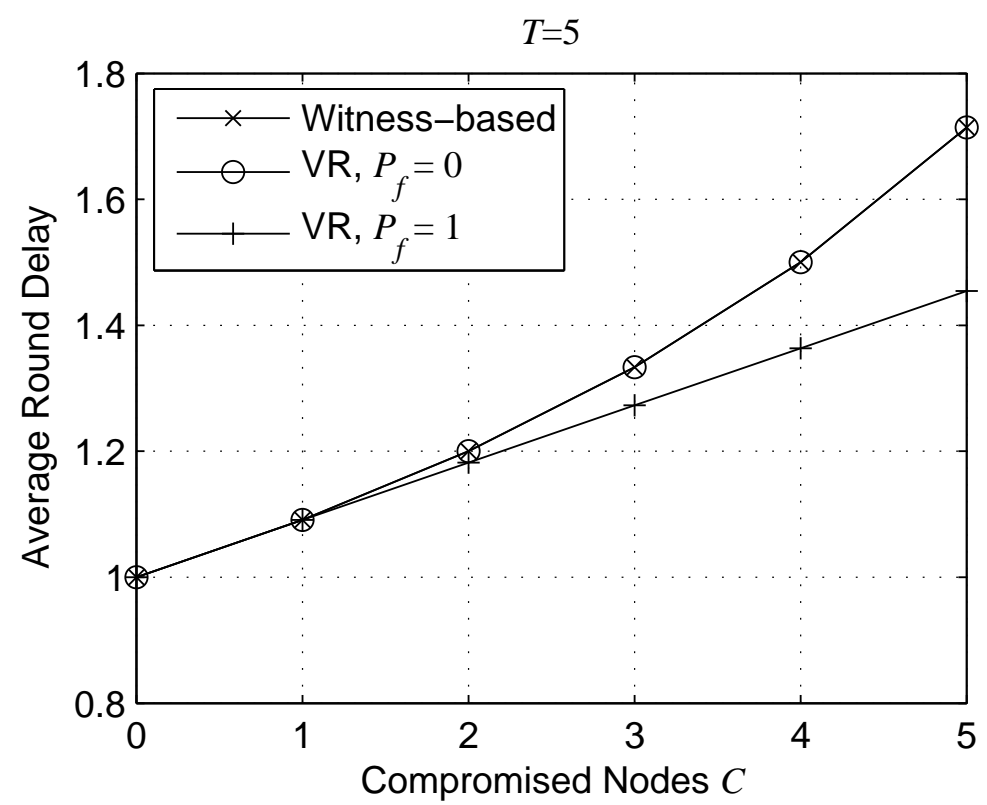

(a)

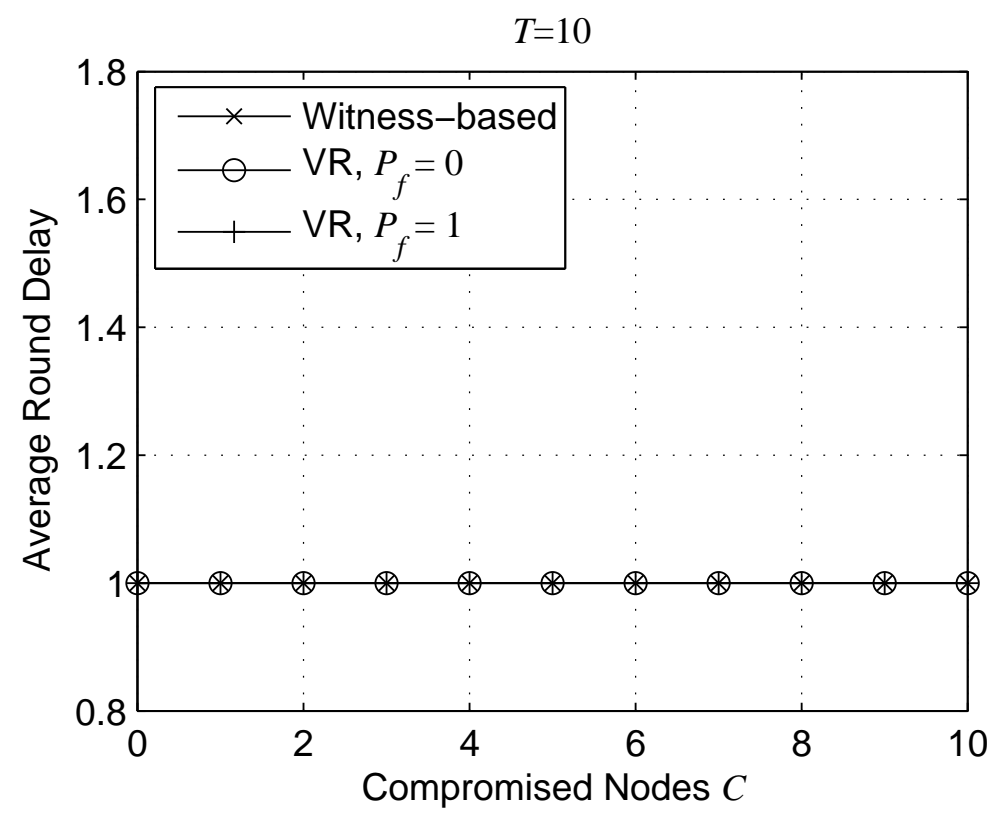

(b)

Fig. 4. Average round delay comparison between the variant-round (VR) scheme and the witness-based approach given in [9], for $M=11$ and $P_{f}=1,0$, (a) when $T=5$ (valid fusion result) and (b) when $T=10$ (invalid fusion result). 


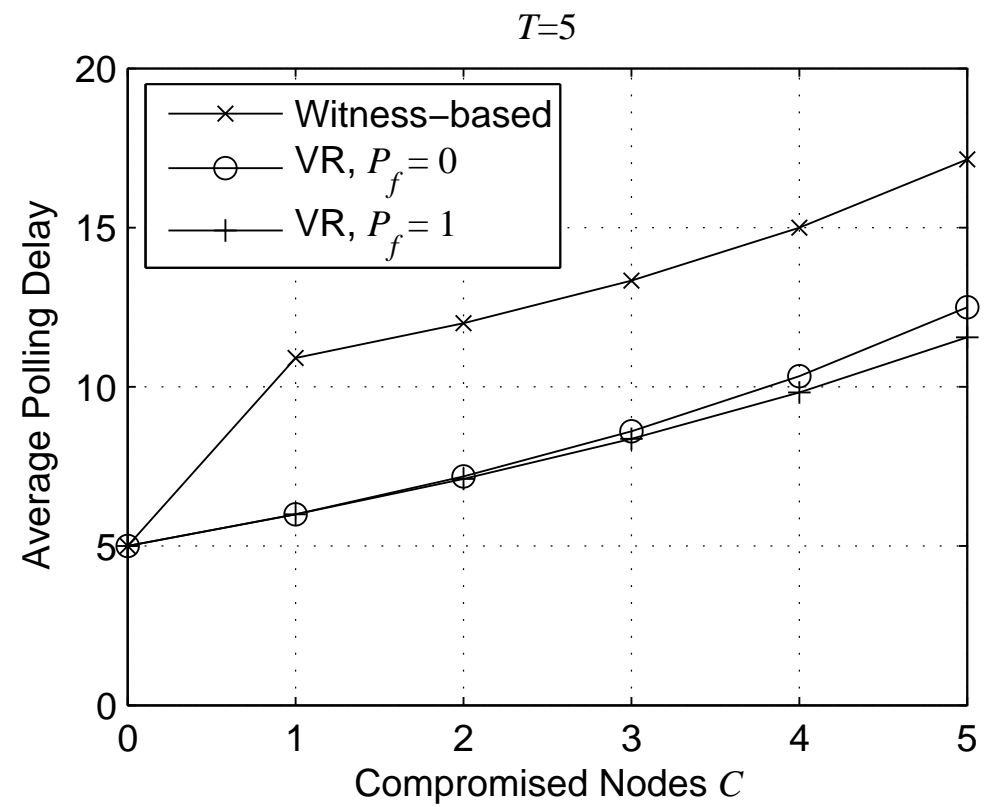

(a)

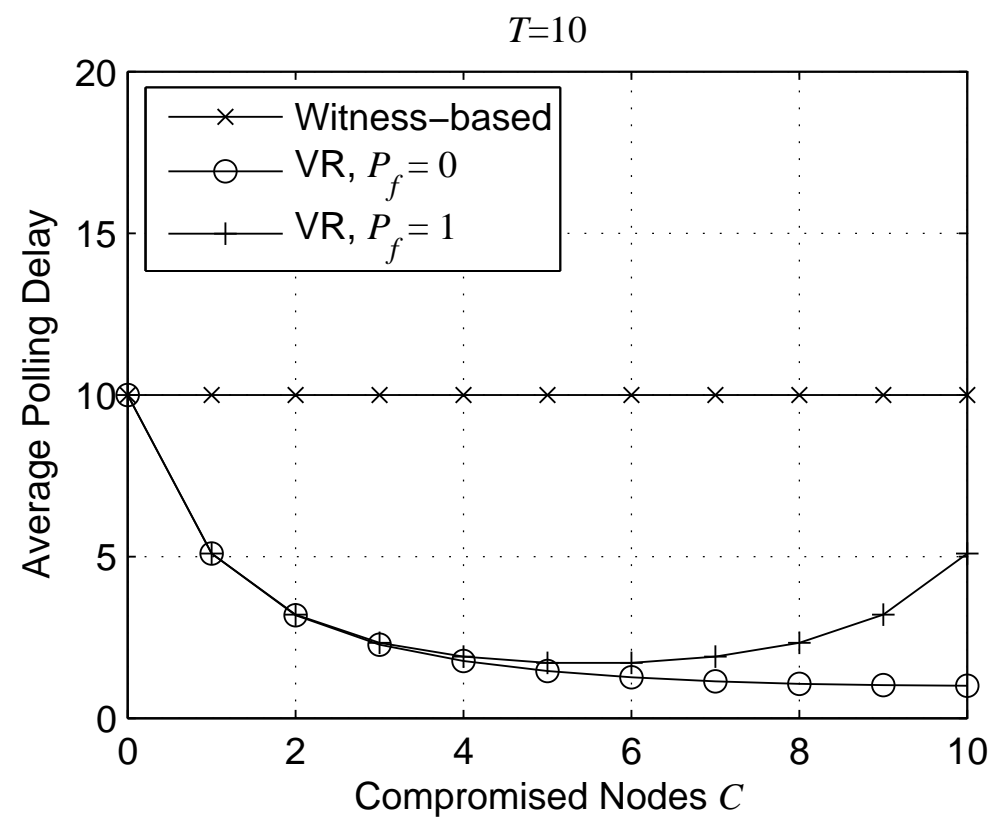

(b)

Fig. 5. Average polling delay comparison between the variant-round (VR) scheme and the witness-based approach given in [9], for $M=11$ and $P_{f}=1,0$, (a) when $T=5$ (valid fusion result) and (b) when $T=10$ (invalid fusion result). 
transmitted by uncompromised nodes in the one-round scheme is about half that of those in the witness-based approach. Additionally, the performance of the one-round scheme when $P_{f}=1$ is the worst in all three simulations, since any compromised node that agrees with the forged result will sometimes make the forged result with the largest number of votes and force the base station to use it as the temporary voting result to poll the next node. Then, the next uncompromised node needs to transmit its fusion result to the base station instead of only sending a agreeing vote and the total number of bits transmitted by the uncompromised nodes increases.

According to Fig. 7(b), when the base station cannot obtain a valid fusion result, the number of bits transmitted by the uncompromised nodes to the base station in the one-round scheme decreases with the number of compromised nodes $C$, except for $P_{f}=1$. This phenomenon is caused by the fact that the scheme stops when the number of unpolled nodes plus the maximum number of votes for any result recorded at the base station is less than $T$. When $T=M-1$ as simulated, the recoding of two different results at the base station stops the polling process. Recall that when $P_{f}=1$, the only way to stop the polling process is for one fusion result to be sent by an uncompromised node and the other to be sent by a compromised node, and for the transmitted bits of the uncompromised nodes to be the same for all $C$. If $P_{f} \neq 1$, the two compromised nodes may yield two different results, and no bit is transmitted by the uncompromised node. This concludes the simulation results. This subfigure reveals that the one-round scheme outperforms when $C$ is small but loses when $C$ exceeds $T / 2$. Similarly, the maximum average number of bits in the one-round scheme is compared with that of the witness-based approach for $M=11$ and 12, and the results are given in Table II. This table indicates that the maximum average number of bits transmitted in the one-round scheme is much lower than that in the witness-based approach.

\section{CONCLUSiOnS AND Future Work}

This work proposes a power-efficient scheme for data fusion assurance, in which the base station in the wireless sensor network collects the fusion data and the votes on the data directly 


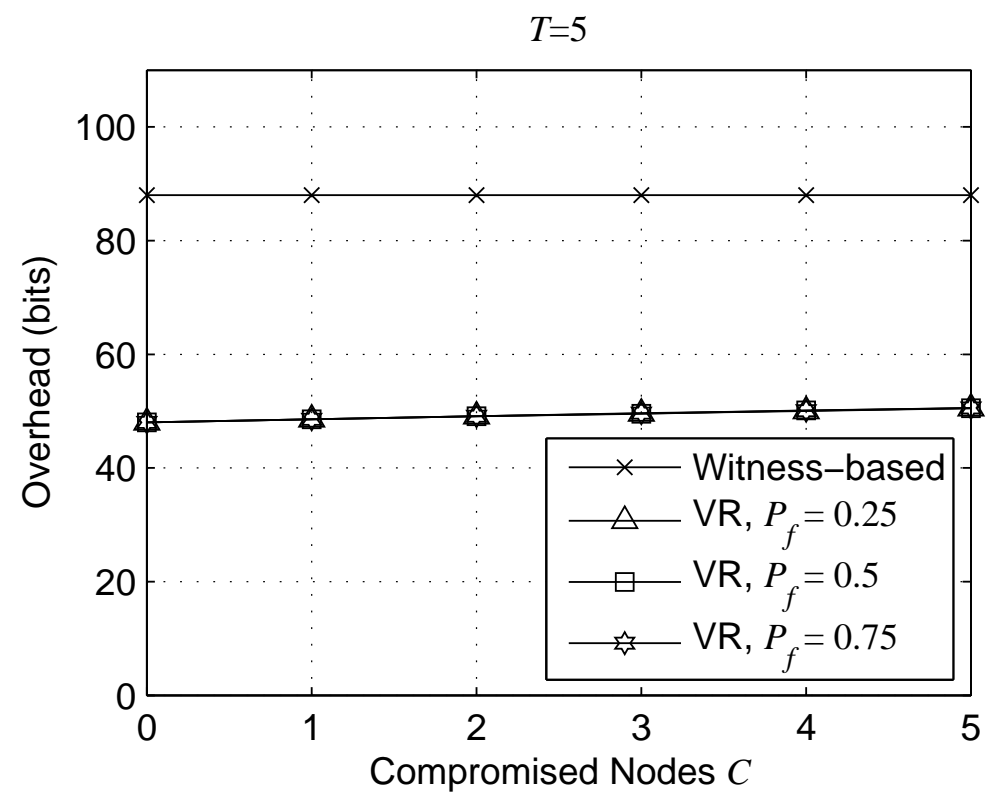

(a)

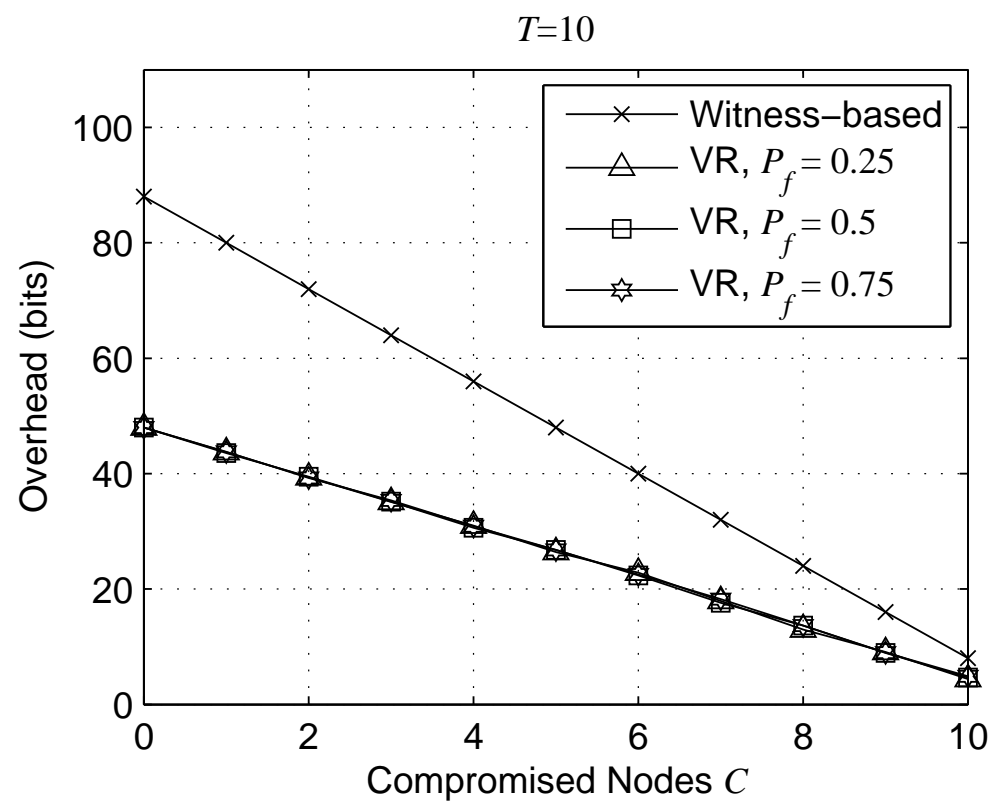

(b)

Fig. 6. Overhead comparison between the variant-round (VR) scheme and the witness-based approach given in [9], for $M=11$ and $P_{f}=0.25,0.5,0.75$, (a) when $T=5$ (valid fusion result) and (b) when $T=10$ (invalid fusion result). 


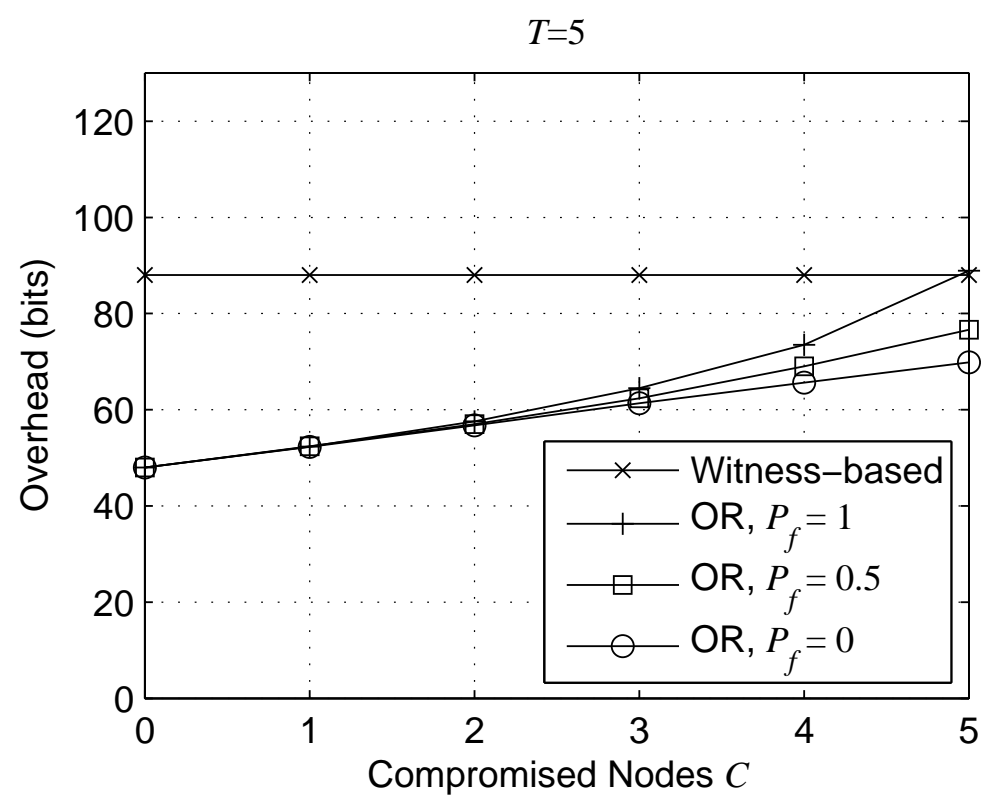

(a)

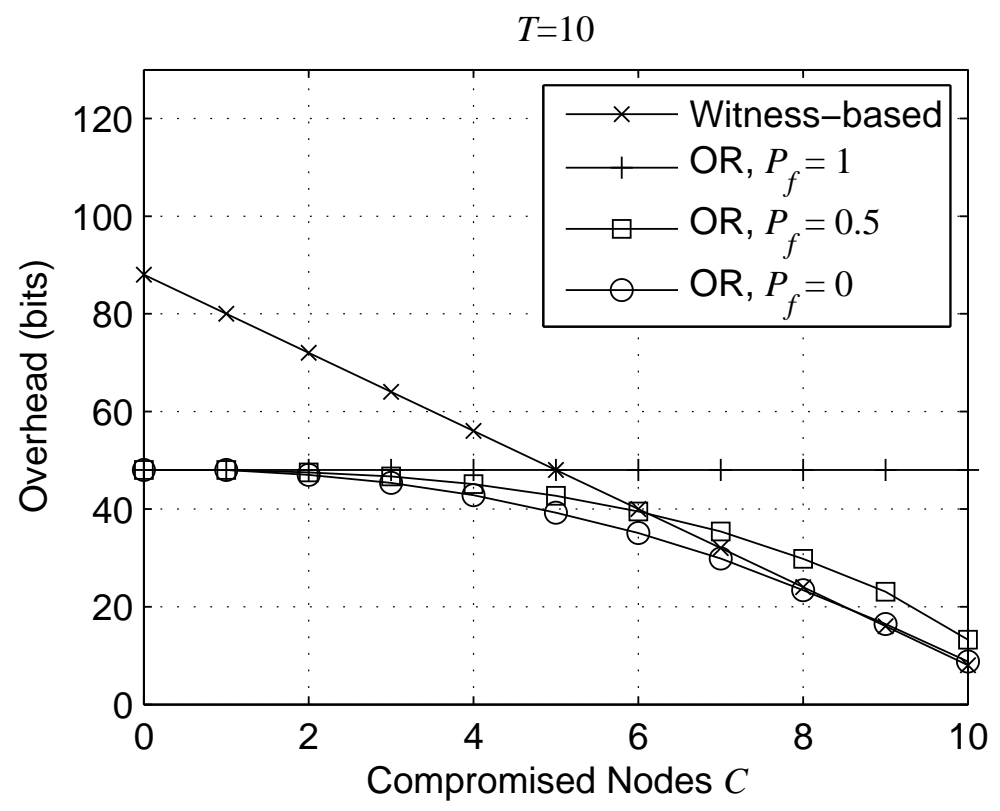

(b)

Fig. 7. Overhead comparison between the one-round (OR) scheme and the witness-based approach given in [9], for $M=11$ and $P_{f}=0,0.5,1$, (a) when $T=5$ (valid fusion result) and (b) when $T=10$ (invalid fusion result). 


\begin{tabular}{|l||c|c|c|c|}
\hline & Witness-based & OR, $P_{f}=0$ & OR, $P_{f}=0.5$ & OR, $P_{f}=1$ \\
\hline$M=11$ & 240 & 71.5 & 86.7 & 128.6 \\
\hline$M=21$ & 566 & 76.1 & 106.4 & 205.7 \\
\hline
\end{tabular}

from the fusion nodes. The proposed scheme is more reliable with less assurance overhead and delay than the witness-based approach. That is, the power and delay for the transmission of the fusion result and the votes are significantly decreased.

In the future, we will discover the performance when a node is compromised with some probability, both statistically dependent and independent to other nodes. Moreover, the propose scheme cannot be applied to multi-hop WSNs. We will develop other schemes based on the direct voting mechanism for the multi-hop WSN.

\section{APPENDIX}

\section{Performance Analysis of the Variant-round Scheme when $P_{f}=1$}

In this analysis we assume that $2 T+1 \geq M$. Since when $C>T$ the base station will get a forged fusion result and this should be avoided, we are only considering two cases:

Case $1 M-T \leq C \leq T$,

Case $2 C<M-T$.

Case 1 does not produce a valid fusion result. Assume that the chosen node at the first round of polling is compromised. The probability that the chosen node is compromised at the first round is given by $C / M$. The first-round polling-for-vote process finishes when $M-T$ witness nodes do not agree with the transmitted fusion result, as described in Step 3. When the polling stops at the $i$ th witness node, the $i$ th witness node (uncompromised) does not agree with the transmitted result and the base station has polled $M-T$ disagreeing nodes (including the $i$ th witness node). Moreover, $M-i-1$ nodes are unpolled, and $M-C-(M-T)=T-C$ of them 
are uncompromised. Since the witness set contains $C-1$ compromised nodes, the maximum number of polled witness nodes is $(C-1)+(M-T)=M-T+C-1$. Hence, the probability that the polling stops at the $i$ th witness node, where $M-T \leq i \leq M-T+C-1$, is given by

$$
P_{v 1}^{c 1}(i)=\frac{1}{\Pi_{v 1}^{c 1}}\left(\begin{array}{c}
i-1 \\
M-T-1
\end{array}\right)\left(\begin{array}{c}
M-i-1 \\
T-C
\end{array}\right) .
$$

The number of agreeing nodes, $A$, equals to $i-(M-T)$. The first witness node that disagreed with the transmitted fusion result at the first round becomes the new chosen node at the second round as stated in Step 4. The size of the witness set becomes $M^{\prime}=(M-1)-(i-M+T)-1=$ $2 M-T-i-2$. If $M^{\prime}<T$, which is equivalent to $(M-1)-T-1<A$, then the polling stops as described in the second part of Step 4. Otherwise, since the previous chosen fusion node is compromised, the current chosen fusion node is not compromised. At the second round, the witness set now has $C^{\prime}=C-1-(i-(M-T))=M+C-T-i-1$ compromised nodes and $M-C-1$ uncompromised nodes. As implied in Step 3, the base station first polls the $M-T-1$ witness nodes that disagreed with the previous transmitted fusion result in the first round. The witness set contains $M-i-1$ unpolled nodes of which $M-C-(M-T)=T-C$ are uncompromised. Consequently, the number of the possible polling orders is given by

$$
\Pi_{v 1}^{c 2}=\left(\begin{array}{c}
M-i-1 \\
T-C
\end{array}\right) .
$$

When the second round of polling stops at the $j$ th witness node, the $j$ th witness node (which is a compromised node) does not agree with the transmitted result. The base station has polled $M^{\prime}-T+1$ disagreeing nodes (including the $j$ th witness node) and $j-\left(M^{\prime}-T+1\right)=j-M^{\prime}+T-1$ agreeing (uncompromised) nodes. The first $M-T-1$ of the $j$ polled nodes are uncompromised, and the rest $j-(M-T-1)-1$ nodes (including the $j$ th witness node) may include the uncompromised or compromised nodes. Since the $j$ polled nodes include $M^{\prime}-T+1$ disagreeing (compromised) nodes, the $M^{\prime}-j$ unpolled nodes include $C^{\prime}-\left(M^{\prime}-T+1\right)$ compromised nodes. The probability that the second polling process stops at the $j$ th witness node, where $j \geq\left(M^{\prime}-T+1\right)+(M-T-1)=M^{\prime}+M-2 T$ and $j \leq\left(M^{\prime}-T+1\right)+(M-C-1)=$ 
$M-C+M^{\prime}-T$ (disagreeing nodes and uncompromised nodes), is given by

$$
\begin{aligned}
P_{v 1}^{c 2}(j) & =\frac{1}{\Pi_{v 1}^{c 2}}\left(\begin{array}{c}
j-(M-T-1)-1 \\
M^{\prime}-T
\end{array}\right)\left(\begin{array}{c}
M^{\prime}-j \\
C^{\prime}-\left(M^{\prime}-T+1\right)
\end{array}\right) \\
& =\frac{1}{\Pi_{v 1}^{c 2}}\left(\begin{array}{c}
j-M+T \\
2 M-2 T-i-2
\end{array}\right)\left(\begin{array}{c}
2 M-T-i-j-2 \\
T+C-M
\end{array}\right) .
\end{aligned}
$$

Since $A$ is at least $M-T-1$ at the second round, it is easy to see that $M^{\prime}-T-1<A$ and the scheme will stop after the second round. Therefore, the average overhead is

$$
O_{v 1}^{c}(M, T, C, 1)=\sum_{i=M-T}^{M-T+C-1} P_{v 1}^{c 1}(i)\left[(M-T) k^{\prime}+\sum_{j=M^{\prime}+M-2 T}^{M-C+M^{\prime}-T} P_{v 1}^{c 2}(j)\left(j-M^{\prime}+T-1\right) k\right] .
$$

As mentioned before, when $M^{\prime}<T$, i.e., $2 M-2 T-2<i$, the scheme will stop after the first round. Hence, the average round delay is

$$
R_{v 1}^{c}(M, T, C, 1)=\sum_{i=M-T}^{2 M-2 T-2} 2 P_{v 1}^{c 1}(i)+\sum_{i=2 M-2 T-1}^{M-T+C-1} P_{v 1}^{c 1}(i),
$$

and the average polling delay is

$$
D_{v 1}^{c}(M, T, C, 1)=\sum_{i=M-T}^{2 M-2 T-2} P_{v 1}^{c 1}(i)\left[i+\sum_{j=M^{\prime}+M-2 T}^{M-C+M^{\prime}-T} j P_{v 1}^{c 2}(j)\right]+\sum_{i=2 M-2 T-1}^{M-T+C-1} i P_{v 1}^{c 1}(i) .
$$

In the first case, the probability that the chosen node is not compromised at the first round is given by $(M-C) / M$. The number of the possible polling orders is written as

$$
\Pi_{v 1}^{u 1}=\left(\begin{array}{c}
M-1 \\
C
\end{array}\right) \text {. }
$$

Thus, the probability that the polling stops at the $i$ th witness node, where $M-T \leq i \leq$ $(M-C-1)+(M-T)=2 M-T-C-1$, is expressed as

$$
P_{v 1}^{u 1}(i)=\frac{1}{\Pi_{v 1}^{u 1}}\left(\begin{array}{c}
i-1 \\
M-T-1
\end{array}\right)\left(\begin{array}{c}
M-i-1 \\
T+C-M
\end{array}\right) .
$$

At the second round the base station chooses the first witness nodes that disagreed with the transmitted fusion result sent in the previous round. The size of the witness set becomes $M^{\prime}=$ $2 M-T-i-2$. The witness set has $M-C-(i-(M-T))-1=2 M-C-T-i-1$ 
uncompromised nodes. Similarly, the polling stops if $M^{\prime}<T$. Otherwise, since the previous chosen fusion node is not compromised, the current chosen fusion node is compromised. Thus, the number of the possible polling orders is given by

$$
\Pi_{v 1}^{u 2}=\left(\begin{array}{c}
M-i-1 \\
T+C-M
\end{array}\right) .
$$

When the second round of polling stops at the $j$ th witness node, the $j$ polled nodes contain $M^{\prime}-T+1$ disagreeing (uncompromised) nodes and the unpolled nodes include $2 M-C-$ $T-i-1-\left(M^{\prime}-T+1\right)$ uncompromised nodes. The probability that the second round of polling finishes at the $j$ th witness node, where $\left(M^{\prime}-T+1\right)+(C-1)=M^{\prime}-T+C \geq j \geq$ $\left(M^{\prime}-T+1\right)+(M-T-1)=M^{\prime}+M-2 T$, is given by

$$
\begin{aligned}
P_{v 1}^{u 2}(j) & =\frac{1}{\Pi_{v 1}^{u 2}}\left(\begin{array}{c}
j-(M-T-1)-1 \\
M^{\prime}-T
\end{array}\right)\left(\begin{array}{c}
M^{\prime}-j \\
2 M-C-T-i-1-\left(M^{\prime}-T+1\right)
\end{array}\right) \\
& =\frac{1}{\Pi_{v 1}^{u 2}}\left(\begin{array}{c}
j-M+T \\
2 M-2 T-i-2
\end{array}\right)\left(\begin{array}{c}
2 M-T-i-j-2 \\
T-C
\end{array}\right) .
\end{aligned}
$$

Similarly, it is easy to see that the scheme will stop after the second round. Therefore, the average overhead is given by

$$
O_{v 1}^{u}(M, T, C, 1)=\sum_{i=M-T}^{2 M-T-C-1} P_{v 1}^{u 1}(i)\left[(i-M+T) k+\sum_{j=M^{\prime}+M-2 T}^{M^{\prime}-T+C} P_{v 1}^{u 2}(j)\left(M^{\prime}-T+1\right) k^{\prime}\right] .
$$

Again, when $M^{\prime}<T$, i.e., $2 M-2 T-2<i$, the scheme will stop after the first round. Hence, the average round delay is

$$
R_{v 1}^{u}(M, T, C, 1)=\sum_{i=M-T}^{2 M-2 T-2} 2 P_{v 1}^{u 1}(i)+\sum_{i=2 M-2 T-1}^{2 M-T-C-1} P_{v 1}^{u 1}(i),
$$

and the average polling delay is

$$
D_{v 1}^{u}(M, T, C, 1)=\sum_{i=M-T}^{2 M-2 T-2} P_{v 1}^{u 1}(i)\left[i+\sum_{j=M^{\prime}+M-2 T}^{M^{\prime}-T+C} j P_{v 1}^{u 2}(j)\right]+\sum_{i=2 M-2 T-1}^{M-T+C-1} i P_{v 1}^{u 1}(i) .
$$

From (8) and (13), we have the average overhead and the average delays of Case 1 as

$$
O_{v 1}(M, T, C, 1)=\frac{C}{M} O_{v 1}^{c}(M, T, C, 1)+\frac{M-C}{M} O_{v 1}^{u}(M, T, C, 1),
$$




$$
R_{v 1}(M, T, C, 1)=\frac{C}{M} R_{v 1}^{c}(M, T, C, 1)+\frac{M-C}{M} R_{v 1}^{u}(M, T, C, 1)
$$

and

$$
D_{v 1}(M, T, C, 1)=\frac{C}{M} D_{v 1}^{c}(M, T, C, 1)+\frac{M-C}{M} D_{v 1}^{u}(M, T, C, 1) .
$$

A valid fusion result is available in the second case. The first-round process is similar to the first-round process in the first case when the chosen node is compromised at the first round. The probability that the $T$ th witness node agrees with the transmitted fusion result at witness node $j$ of the second-round polling process is

$$
\begin{aligned}
P_{v 2}^{c 2}(j) & =\frac{1}{\Pi_{v 1}^{c 2}}\left(\begin{array}{c}
j-(M-T-1)-1 \\
T-(M-T-1)-1
\end{array}\right)\left(\begin{array}{c}
2 M-T-i-j-2 \\
M-C-(T+1)
\end{array}\right) \\
& =\frac{1}{\Pi_{v 1}^{c 2}}\left(\begin{array}{c}
j-M+T \\
2 T-M
\end{array}\right)\left(\begin{array}{c}
2 M-T-i-j-2 \\
M-C-T-1
\end{array}\right) .
\end{aligned}
$$

Significantly, if $M=2 T+1$, then $j=T$. Otherwise, $T \leq j \leq C-(i-(M-T))-1+T=$ $M+C-i-1$. Therefore, the average overhead is expressed as

$$
O_{v 2}^{c}(M, T, C, 1)= \begin{cases}\sum_{i=M-T}^{M-T+C-1} P_{v 1}^{c 1}(i)\left[(M-T) k^{\prime}+T k\right]=(M-T) k^{\prime}+T k & M=2 T+1 \\ \sum_{i=M-T}^{M-T+C-1} P_{v 1}^{c 1}(i)\left[(M-T) k^{\prime}+\sum_{j=T}^{M+C-i-1} P_{v 2}^{c 2}(j) T k\right] & \text { else }\end{cases}
$$

Hence, the average round delay is

$$
R_{v 2}^{c}(M, T, C, 1)=\sum_{i=M-T}^{M-T+C-1} 2 P_{v 1}^{c 1}(i)
$$

and the average polling delay is

$$
D_{v 2}^{c}(M, T, C, 1)=\sum_{i=M-T}^{M-T+C-1} P_{v 1}^{c 1}(i)\left[i+\sum_{j=T}^{M+C-i-1} j P_{v 2}^{c 2}(j)\right] .
$$

Only one round of polling is needed when the chosen node is uncompromised at the first round. The probability that the polling process ends at the witness node $i$, where $T \leq i \leq T+C$, is written as

$$
P_{v 2}^{u 1}(i)=\frac{1}{\prod_{v 1}^{u 1}}\left(\begin{array}{c}
i-1 \\
T-1
\end{array}\right)\left(\begin{array}{c}
M-i-1 \\
M-C-T-1
\end{array}\right)
$$


The average overhead, and the delays are given by

$$
\begin{gathered}
O_{v 2}^{u}(M, T, C, 1)=\sum_{i=T}^{T+C} P_{v 2}^{u 1}(i) T k, \\
R_{v 2}^{u}(M, T, C, 1)=1,
\end{gathered}
$$

and

$$
D_{v 2}^{u}(M, T, C, 1)=\sum_{i=T}^{T+C} i P_{v 2}^{u 1}(i) .
$$

From (17) and (22), the average overhead, the delays in Case 2 are given by

$$
\begin{aligned}
& O_{v 2}(M, T, C, 1)=\frac{C}{M} O_{v 2}^{c}(M, T, C, 1)+\frac{M-C}{M} O_{v 2}^{u}(M, T, C, 1), \\
& R_{v 2}(M, T, C, 1)=\frac{C}{M} R_{v 2}^{c}(M, T, C, 1)+\frac{M-C}{M} R_{v 2}^{u}(M, T, C, 1),
\end{aligned}
$$

and

$$
D_{v 2}(M, T, C, 1)=\frac{C}{M} D_{v 2}^{c}(M, T, C, 1)+\frac{M-C}{M} D_{v 2}^{u}(M, T, C, 1)
$$

\section{REFERENCES}

[1] I. F. Akyildiz, W. Su, Y. Sankarasubramaniam, and E. Cayirci, "Wireless sensor networks: a survey," IEEE Commun. Mag., vol. 40, pp. 102-114, Aug. 2002.

[2] S. A. Aldosari and J. M. F. Moura, "Detection in decentralized sensor networks," in Proc. ICASSP 2004, Montreal, Canada, May 2004.

[3] R. Anderson and M. Kuhn, "Tamper resistance - a cautionary note," in Proc. 2nd Useix Workshop on Electronic Commerce, Oakland, CA, Nov. 1996, pp. 1-11.

[4] J.-F. Chamberland and V. V. Veeravalli, "Aymptotic results for decentralized detection in power constrained wireless sensor networks," IEEE J. Select. Areas Commun., vol. 22, no. 6, pp. 1007-1015, Aug. 2004.

[5] D. Culler, D. Estrin, and M. Srivastava, "Overview of sensor networks," IEEE Computer, vol. 37, no. 8, pp. 41-49, Aug. 2004.

[6] L. Dan, K. D. Wong, H. H. Yu, and A. M. Sayeed, "Detection, classification, and tracking of targets," IEEE Trans. Signal Processing, vol. 19, no. 3, pp. 17-29, Mar. 2002.

[7] A. D'Costa, V. Ramachandran, and A. M. Sayeed, "Distributed classification of gaussian space-time sources in wireless sensor networks," IEEE J. Select. Areas Commun., vol. 22, no. 6, pp. 1026-1036, Aug. 2004.

[8] J. Deng, R. Han, and S. Mishra, "A performance evaluation of intrusion-tolerant routing in wireless sensor networks," in Proc. IPSN 2003, Palo Alto, CA, 2003, pp. 349-364.

[9] W. Du, J. Deng, Y. S. Han, and P. K. Varshney, "A witness-based approach for data fusion assurance in wireless sensor networks," in Proc. GLOBECOM 2003, vol. 3, San Francisco, CA, Dec. 2003, pp. 1435-1439. 
[10] Y. Lin, B. Chen, and P. K. Varshney, "Decision fusion rules in multi-hop wireless sensor networks," IEEE Trans. Aerosp. Electron. Syst., vol. 41, no. 2, pp. 475-488, Apr. 2005.

[11] J. N. Al-Karaki and A. E. Kamal, "Routing techniques in wireless sensor networks: a survey," IEEE Wireless Commun. Mag., vol. 11, no. 6, pp. 6-28, Dec. 2004.

[12] D. Niculescu, "Positioning in ad hoc sensor networks," IEEE Network, vol. 18, no. 4, pp. 24-29, Jul.-Aug. 2004.

[13] —_, "Communication paradigms for sensor networks," IEEE Commun. Mag., vol. 43, no. 3, pp. 116-122, Mar. 2005.

[14] S. Olariu, A. Wadaa, L. Wilson, and M. Eltoweissy, "Wireless sensor networks: leveraging the virtual infrastructure," IEEE Network, vol. 18, no. 4, pp. 51-56, Apr. 2004.

[15] S. Olariu and Q. Xu, "Information assurance in wireless sensor networks," in Proc. IEEE IPDPS'05, Denver, CO, Apr. 2005, pp. 236a-236a.

[16] A. Perrig, J. A. Stankovic, and D. Wagner, "Security in wireless sensor networks," Communications of the ACM, vol. 47, no. 6, pp. 53-57, June 2004.

[17] B. Przydatek, D. Song, and A. Perrig, "SIA: secure information aggregation in sensor networks," in Proc. SenSys 2003, Los Angeles, CA, Nov. 2003, pp. 255-265.

[18] R. C. Shah, S. Roy, S. Jain, and W. Brunette, "Data mules: modeling a three-tier architecture for sparse sensor networks," in Proc. IEEE Workshop on Sensor Network Protocols and Applications, Anchorage, AK, May 2003, pp. 30-41.

[19] C.-C. Shen, C. Srisathapornphat, and C. Jaikaeo, "Sensor information networking architecture and applications," IEEE Personal Commun. Mag., vol. 8, no. 4, pp. 52-59, Aug. 2001.

[20] P. Sholander, A. Harris, and J. Brown, "Intersensor information assurance for dod tactical networks," in Proc. MILCOM 2002, vol. 2, Anaheim, CA, Oct. 2002, pp. 1456-1461.

[21] F. Sivrikaya and B. Yener, "Time synchronization in sensor networks: a survey," IEEE Network, vol. 18, no. 4, pp. 45-50, Jul.-Aug. 2004.

[22] K. Sohrabi, W. Merrill, J. Elson, L. Girod, F. Newberg, and W. Kaiser, "Methods for scalable self-assembly of ad hoc wireless sensor networks," IEEE Trans. Mobile Comput., vol. 3, no. 4, pp. 317-331, Oct.-Dec. 2004.

[23] L. Tong, Q. Zhao, and S. Adireddy, "Sensor networks with mobile agents," in Proc. IEEE MILCOM'03, Boston, MA, Oct. 2003, pp. 688-693.

[24] J. N. Tsitsiklis, "Decentralized detection by a large number of sensors," Mathematics of Conrol, Signals, and Systems, vol. 1, no. 2, pp. 167-182, 1988.

[25] P. K. Varshney, Distributed Detection and Data Fusion. New York: Springer, 1997.

[26] T.-Y. Wang, Y. S. Han, and P. K. Varshney, "A combined decision fusion and channel coding scheme for fault-tolerant classification in wireless wensor networks," in Proc. ICASSP 2004, Montreal, Canada, May 2004.

[27] T.-Y. Wang, Y. S. Han, P. K. Varshney, and P.-N. Chen, "Distributed fault-tolerant classification in wireless sensor networks," IEEE J. Select. Areas Commun., vol. 23, no. 4, pp. 724-734, Apr. 2005.

[28] Z. Yang and L. Tong, "Cooperative sensor networks with misinformed nodes," IEEE Trans. Inform. Theory, vol. 51, no. 12, pp. 4118-4133, Dec. 2005.

[29] W. Zhao and M. H. Ammar, "Message ferrying: proactive routing in highly-partitioned wireless ad hoc networks," in Proc. IEEE Workshop on Future Trends in Distributed Computing Systems, Puerto Rico, May 2003, pp. 308-314. 
